\title{
NONORIENTABLE SURFACES IN SOME NON-HAKEN 3-MANIFOLDS
}

BY

\author{
J. H. RUBINSTEIN
}

\begin{abstract}
If a closed, irreducible, orientable 3-manifold $M$ does not possess any 2-sided incompressible surfaces, then it can be very useful to investigate embedded one-sided surfaces in $M$ of minimal genus. In this paper such 3-manifolds $M$ are studied which admit embeddings of the nonorientable surface $K$ of genus 3 . We prove that a 3-manifold $M$ of the above type has at most 3 different isotopy classes of embeddings of $K$ representing a fixed element of $H_{2}\left(M, Z_{2}\right)$. If $M$ is either a binary octahedral space, an appropriate lens space or Seifert manifold, or if $M$ has a particular type of fibered knot, then it is shown that the embedding of $K$ in $M$ realizing a specific homology class is unique up to isotopy.
\end{abstract}

1. Introduction. All manifolds and maps will be assumed to be PL. Let $K$ denote a closed nonorientable surface of genus 3 , i.e. $K$ is the connected sum of 3 copies of $R P^{2}$. Our aim is to study the embeddings of $K$ into some irreducible 3-manifolds $M$ which are non-Haken, i.e. do not possess any properly embedded 2-sided incompressible surfaces. Such an embedding of $K$ can play the role of a hierarchy, as in Waldhausen's work on Haken 3-manifolds [12] and the results obtained here will be used in [2] to calculate the homeotopy groups of the 3-manifolds $M$.

Definition (SEE [8]). Let $M$ be a closed orientable 3-manifold. $M$ is said to have a one-sided Heegaard splitting of genus 3 if $M=N(K) \cup Y$, where $N(K)$ is an orientable line-bundle over $K, Y$ is a handlebody of genus 2 and $N(K) \cap Y=$ $\partial N(K)=\partial Y$.

The fundamental result in this paper is as follows: Let $M$ be a closed orientable irreducible atoroidal 3-manifold $\neq R P^{3}$ which has a one-sided Heegaard splitting of genus 3, $M=N(K) \cup Y$. Suppose that $K^{\prime} \subset M$ is another embedding of the genus 3-nonorientable surface in $M$, with the property that $K$ and $K^{\prime}$ represent the same class in $H_{2}\left(M, Z_{2}\right)$. Then $K^{\prime}$ is isotopic to $K^{\prime \prime}$ so that $K \cap K^{\prime \prime}$ is a single curve.

As a corollary to the above theorem, it is established that if $M \neq R P^{3}$ is closed, orientable, irreducible and non-Haken, and $M$ admits an embedding of $K$, then there are at most 3 different isotopy classes of embeddings of $K$ realizing a particular element of $H_{2}\left(M, Z_{2}\right)$. More precisely, suppose $K \subset M$ is an embedding and let $|K|$ denote the image of the generator of $H_{2}\left(K, Z_{2}\right)$ in $H_{2}\left(M, Z_{2}\right)$ under the map induced by the inclusion $K \subset M$. Then there are at most two other embeddings $K^{\prime}$,

Received by the editors April 28, 1980 and, in revised form, February 27, 1981.

1980 Mathematics Subject Classification. Primary 57N10, 57Q37, 57R95.

Key words and phrases. Non-Haken 3-manifold, one-sided Heegaard splitting, isotopy class of embeddings, genus 3 nonorientable surface. 
$K^{\prime \prime} \subset M$ of the genus 3 nonorientable surface in $M$ with $\left|K^{\prime}\right|=\left|K^{\prime \prime}\right|=|K|$, such that if $K^{*} \subset M$ is any other embedding with $\left|K^{*}\right|=|K|$, then $K^{*}$ is isotopic to one of $K, K^{\prime}$ or $K^{\prime \prime}$.

It turns out that $K^{\prime}, K^{\prime \prime}$ can be chosen so that each of $K \cap K^{\prime}, K \cap K^{\prime \prime}$ and $K^{\prime} \cap K^{\prime \prime}$ is the same single curve. We are unable to determine if there is ever a genuine obstruction or not to isotoping $K^{\prime}$ and $K^{\prime \prime}$ to $K$ (see §7).

Finally the useful theorem is obtained that for a large number of special choices of $M$, there is a single isotopy class of embeddings of $K$ realising a fixed element of $H_{2}\left(M, Z_{2}\right)$. The examples of such $M$ include all the binary octahedral spaces, the lens spaces of type $L(8 p q+2 \varepsilon, 4 p q \pm 2 p+\varepsilon)$ where $\varepsilon= \pm 1, p, q \geqslant 1$ (cf. Theorem 12 of [18]), various non-Haken Seifert manifolds with infinite fundamental group, and some 3-manifolds which contain certain types of fibered knots, e.g. the result of certain surgeries on the figure 8 knot. In [10] and [5], it is shown that many of the latter examples of 3-manifolds are non-Haken.

This paper can be viewed as a sequel to [9], in which the same program was carried out for embeddings of the Klein bottle in some closed orientable non-Haken 3-manifolds.

2. Examples of 3-manifolds. We will be concerned with the following 3 classes of closed, orientable, irreducible 3-manifolds which admit embeddings of $K$ and are non-Haken:

(1) The closed orientable Seifert manifolds with $S^{2}$ as orbit surface which have 3 exceptional fibres of multiplicity $(2,1),(4 k, 2 k-1),(m, n)$ and arbitrary $b$-invariant, where $k \geqslant 1$ and $m \geqslant 2$ (see [7] for Seifert manifold notation). These are irreducible and non-Haken, so long as the cases where $(m, n)=(4 k, 1)$ and $b=-1$ are excluded. Also we include in this class the lens spaces of the form $L(8 p q+2 \varepsilon$, $4 p q \pm 2 p+\varepsilon)$, where $p, q \geqslant 1$ and $\varepsilon= \pm 1$. These are precisely the lens spaces which admit embeddings of $K$ (see [4]) and can also be characterized as the closed orientable Seifert manifolds with $S^{2}$ as orbit surface which have 2 exceptional fibres of multiplicity $(2,1),(4 k, 2 k-1)$ and arbitrary $b$-invariant (see pp. 99-100 of [7]).

Remark. The closed orientable Seifert manifolds $M$ which have $S^{2}$ as orbit surface, 3 exceptional fibres of multiplicity $(2,1),(4,1),(3, \pm 1)$ and arbitrary $b$-invariant, all have $\pi_{1}(M)$ equal to a finite group of the form $O(48) \times Z_{n}$, where $O(48)$ is the binary octahedral group. These 3-manifolds are often called the binary octahedral spaces (see Chapter 6 of [7]).

(2) The closed orientable Seifert manifolds with $S^{2}$ as orbit surface which have 3 exceptional fibres of multiplicity $(r, \pm 2),(s, \pm 2),(t, \pm 2)$ and $b$-invariant equal to zero, where $r, s, t$ are odd integers $\geqslant 3$.

REMARK. The lens spaces in class (1) can also be represented as the closed orientable Seifert manifolds with $S^{2}$ as orbit surface and 2 exceptional fibres of multiplicity $(r, \pm 2),(s, \pm 2)$, with $b$-invariant equal to \pm 2 (see pp. $99-100$ of [7]). So these lens spaces can be included in either category (1) or (2).

(3) The closed orientable 3-manifolds $M \neq R P^{3}$ which are irreducible, non-Haken and have one-sided Heegaard splittings of genus 3, with the following extra condition $(*)$ satisfied. 
Let $C$ be a simple closed curve on $K$ such that $K-C$ is orientable (i.e. a punctured torus). Since $N(K)$ is homeomorphic to the mapping cylinder of the orientable double covering of $K$, we may regard $\partial N(K)$ as the orientable double covering of $K$. Let $C^{\prime}$ denote the lift of $C$ to $\partial N(K)$. The condition (*) is that there is some meridian disk $D$ for the handlebody $Y$, in the decomposition $M=N(K) \cup Y$, with the property that $\partial D$ intersects $C^{\prime}$ transversely in exactly two points.

REMARKS. (a) The condition (*) is satisfied by all the closed orientable 3-manifolds which are formed by glueing a solid torus to a fibre bundle $X$ over $S^{1}$ with fibre a punctured torus, so that the meridian curve of the solid torus runs twice along the base circle of the bundle (see §3). In [5] it is shown that most such 3-manifolds $M=X \cup B^{2} \times S^{1}$ are irreducible and non-Haken.

(b) The compiement of the figure $8 \mathrm{knot}$ in $S^{3}$ is a punctured torus bundle over $S^{1}$. So $(2,2 n+1)$ surgery on this knot gives a 3-manifold $M=X \cup B^{2} \times S^{1}$ as in Remark (a) above. In [10] it is proved that, for $n \neq 0, M$ is irreducible, non-Haken and hyperbolic, whereas for $n=0$ the surgery yields a Seifert manifold as in class (1) above.

\section{Properties of the 3-manifolds.}

LEMMA 1. The 3-manifolds in classes (1) and (2) are irreducible and non-Haken.

Proof. The irreducibility of the examples follows by [11]. Also by [11], closed orientable Seifert manifolds $M$ with $S^{2}$ as orbit surface and 3 exceptional fibres are non-Haken if and only if $H_{1}(M, Z)$ is finite.

For $M$ in class (1), the relation matrix for $H_{1}(M, Z)$ has determinant $=$ $-(8 b k m+8 k n+2 m(2 k-1)+4 k m)$. So $H_{1}(M, Z)$ is infinite exactly when $4 b k m$ $+4 k n+4 k m-m=0$. But $m$ and $n$ are relatively prime, so $m=4 k$ and $n=1-$ $m(1+b)$. However $0<n<m$ and consequently $b=-1, n=1$ are the only solutions.

For $M$ in class (2), the relation matrix for $H_{1}(M, Z)$ has determinant $= \pm 2 r s \pm$ $2 r t \pm 2 s t$. But $r, s, t$ are all odd and so $\pm r s \pm r t \pm s t$ is always odd. Hence $H_{1}(M, Z)$ is always finite.

LEMMA 2. The 3-manifolds in $\$ 2$ all admit embeddings of $K$.

Proof. Suppose $M$ is of type (1). Let $N_{1}$ and $N_{2}$ be small fibered neighborhoods of the exceptional fibres of multiplicity 2 and $4 k$ respectively. It is easy to see that an ordinary fibre in $\partial N_{1}$ bounds a Möbius band $J$ in $N_{1}$.

On the other hand, let $h$ be an ordinary fibre in $\partial N_{2}$. If a solid torus $N$ is glued to $N_{2}$ so that a meridian curve for $N$ is matched up with $h$, then the result is the lens space $L(4 k, 2 k-1)$. In [9] it is shown that such a lens space contains a Klein bottle, which can be chosen to meet $N$ in a single meridian disk. Consequently we obtain that $h$ bounds a punctured Klein bottle $L$ in $N_{2}$.

Finally there is an annulus $A$ of ordinary fibres in $M-$ int $N_{1}-$ int $N_{2}$ with $\partial A=\partial J \cup \partial L$. Hence $J \cup A \cup L=K$ is the desired genus 3 nonorientable surface embedded in $M$. 
Next, assume $M$ is in the class (2). Let $N_{i}, i=1,2,3$, be small fibered neighborhoods of the 3 exceptional fibres. Then there is a homeomorphism $\psi: E \times S^{1} \rightarrow M$ $-\cup_{i}$ int $N_{i}$, where $S^{1}=\{z \in \mathbf{C}:|z|=1\}$ and $E$ is a 2 -sphere with 3 punctures. $E$ can be chosen so that the curve $C_{i}=\psi(E \times\{1\}) \cap \partial N_{i}$ intersects an ordinary fibre $h_{i}$, lying in $\partial N_{i}$, transversely in one point. Then the meridian curves for $N_{i}$ have homology classes in $H_{1}\left(\partial N_{i}, Z\right)$ given by $r\left|C_{1}\right| \pm 2\left|h_{1}\right|, s\left|C_{2}\right| \pm 2\left|h_{2}\right|, t\left|C_{3}\right| \pm 2$ $\left|h_{3}\right|$, where $|x|$ denotes the homology class of $x$. Note that this uses the fact that the $b$-invariant for $M$ is zero and the other invariants are $(r, \pm 2),(s, \pm 2),(t, \pm 2)$.

Finally since the intersection number of $C_{i}$ with the meridian curve for $N_{i}$ is \pm 2 , it follows that $C_{i}$ bounds a Möbius band $J_{i}$ in $N_{i}$ for $i=1,2,3$. Therefore $K=$ $\psi(E \times\{1\}) \cup J_{1} \cup J_{2} \cup J_{3}$ is the desired genus 3 nonorientable surface embedded in $M$.

Finally let $M$ be a 3-manifold as in Remark (a), following the description of class (3). We prove that $M$ has a one-sided Heegaard splitting of genus 3 which satisfies condition (*). Let $X$ be a fibre bundle over $S^{1}$ with fibre a punctured torus. Let $N$ be a solid torus and let $M=X \cup N$, where $N$ is glued to $X$ so that a meridian curve for $N$ has homology class in $H_{1}(\partial X, Z)$ given by $2 t+(2 n+1) u$. Here $u$ is the homology class of $\partial T_{0}$, where $T_{0}$ is some fixed fibre of $X$, and $\{t, u\}$ form a generating set for $H_{1}(\partial X, Z)$.

Exactly as before, $\partial T_{0}$ bounds a Möbius band $J$ in $N$ and so $K=T_{0} \cup J$ is the required genus 3 nonorientable surface. Let $S^{1}=\{z \in \mathbf{C}:|z|=1\}$ and let $I_{+}=$ $\left\{z \in S^{1}: 0 \leqslant \arg z \leqslant \pi\right\}, I_{-}=\left\{z \in S^{1}: \pi \leqslant \arg z \leqslant 2 \pi\right\}$. Let $p: X \rightarrow S^{1}$ be the bundle projection and let $Y_{+}=p^{-1}\left(I_{+}\right), Y_{-}=p^{-1}\left(I_{-}\right)$. Then it is clear that we can choose $N(K)=Y_{+} \cup N$ and $Y=Y_{-}$. There are homeomorphisms $\phi_{1}: T_{0} \times$ $[0, \pi] \approx Y_{+}$and $\phi_{2}: T_{0} \times[\pi, 2 \pi] \approx Y_{-}$.

A centre-line $C$ of the Möbius band $J$ is a core circle of the solid torus $N$. Hence the lift $C^{\prime}$ of $C$ to $\partial N(K)=\partial\left(Y_{+} \cup N\right)$ can be taken as the curve $\phi_{1}\left(\partial T_{0} \times\{\pi\}\right)$. But a meridian disk $D$ for $Y=Y_{-} \approx T_{0} \times[\pi, 2 \pi]$ can be chosen to be $\phi_{2}(\lambda \times[\pi, 2 \pi])$, where $\lambda$ is any arc which is properly embedded in $T_{0}$ and is not homotopic keeping its ends fixed into $\partial T_{0}$. It is immediate that $\partial D \cap C^{\prime}$ consists of two points and so condition (*) is fulfilled.

REMARKS. (a) It is easy to check that $M$ can be decomposed as $X \cup N$, where $X$ is a punctured torus bundle over $S^{1}$ and a meridian disk for $N$ has homology class of the form $2 t+(2 n+1) u$, if and only if $M$ has a one-sided Heegaard decomposition $M=N(K) \cup Y$ such that $Y$ has meridian disks $D_{1}, D_{2}$ for which $Y-D_{1}-D_{2}$ is simply connected and $\partial D_{i} \cap C^{\prime}$ consists of 2 points, for $i=1,2$.

(b) Combining the results of Lemmas 1 and 2 with Lemma 2 of [8], we obtain that all the 3-manifolds in classes (1) and (2) have one-sided Heegaard splittings of genus 3.

4. The main theorem. We begin with the following important result on the behaviour of genus 3 nonorientable surfaces.

Lemma 3. Suppose $C_{1}, C_{2}$ are simple closed curves in $K$ such that both $K-C_{1}$ and $K-C_{2}$ are orientable. Then $C_{1}$ is isotopic to $C_{2}$ in $K$. 
Proof. This follows by Theorem 3 of [1], since all the generators of the mapping class group of $K$ preserve the isotopy class of $C_{1}$, and there is clearly a homeomorphism of $K$ taking $C_{1}$ to $C_{2}$.

Corollary 4. The mapping class group of $K$ is isomorphic to $\mathrm{GL}(2, Z)$. In fact, the representation of homeomorphisms of $K$ by their induced action on the free part of $H_{1}(K, Z)$ gives this isomorphism.

Proof. This is established either by using Lemma 3 and noting that $K-C_{1}$ is a punctured torus, or directly by the presentation of the mapping class group given in Theorem 3 of [1].

REMARK. A direct proof of Lemma 3 can be given by a similar argument to [1].

THEOREM 5. Assume that $M$ is a closed, orientable, irreducible, atoroidal 3-manifold $\neq R P^{3}$ and that $M$ has a genus 3 one-sided Heegaard decomposition, $M=N(K) \cup Y$. Let $K^{\prime}$ be another embedding of a genus 3 nonorientable surface in $M$ with $|K|=\left|K^{\prime}\right|$. Then $K^{\prime}$ is isotopic to $K^{\prime \prime}$ so that $K \cap K^{\prime \prime}$ is single curve $C$ and both $K-C$ and $K^{\prime \prime}-C$ are orientable.

ReMARK. This result probably fails without the assumption that $M$ is atoroidal. In fact, if $M$ contains incompressible tori then the homeotopy group of $M$ can contain elements of infinite order given by "Dehn twists" about such tori. Applying such a homeomorphism to $K$, we can obtain an embedding $K^{\prime}$ for which $K \cap K^{\prime}$ contains many curves and there is no obvious way to decrease the number of components of $K \cap K^{\prime}$ by an isotopy of $K^{\prime}$ (cf. the proof of Theorem 5).

Proof. If $K$ is geometrically compressible, then since $M$ is irreducible it follows that $M=R P^{3}$ and $K$ is just $R P^{2}$ with a trivial handle added. We exclude this case; otherwise $K^{\prime}$ could be $R P^{2}$ with a very knotted handle attached. So we have that $K$ and $K^{\prime}$ are geometrically incompressible in $M$. Assume that $K$ intersects $K^{\prime}$ transversely. If some simple closed curve of $K \cap K^{\prime}$ contracts in $K$ then it must contract in $K^{\prime}$ and vice versa. Because $M$ is irreducible it is easy to find an isotopy of $K^{\prime}$ which eliminates all such contractible curves in $K \cap K^{\prime}$. Consequently we may suppose that $K$ and $K^{\prime}$ intersect transversely in loops which are noncontractible in both $K$ and $K^{\prime}$, without loss of generality.

Next, $N(K)$ can be shrunk so that $K^{\prime} \cap N(K)$ consists of annuli and Möbius bands, each meeting $K$ in a single noncontractible curve. Also any orientationreversing curve on $K^{\prime}$ has intersection number one mod 2 with $K^{\prime}$ (Poincare duality with $Z_{2}$ coefficients). As $|K|=\left|K^{\prime}\right|$, such a curve must intersect $K$. We conclude that $K^{\prime}-K$ and $K^{\prime} \cap Y$ are both orientable.

It is easy to see that all the components of $K^{\prime} \cap Y$ must be annuli except for one component which is either a torus with one puncture or a sphere with 3 holes. By symmetry the same is true for the components of $K-$ int $N\left(K^{\prime}\right)$, where $N\left(K^{\prime}\right)$ is a small regular neighborhood of $K^{\prime}$. Note that all the components of $K^{\prime} \cap Y$ are (2-sided) incompressible surfaces in $Y$, since $K^{\prime}$ is geometrically incompressible.

If any annulus of $K^{\prime} \cap Y$ is parallel into $\partial Y$, then it can be eliminated by an isotopy of $K^{\prime}$. On the other hand, if such an annulus $A$ is not boundary-parallel, 
then it is easy to show that there is a boundary compression of $A$ which produces a meridian disk $D$ of $Y$. $D$ can be chosen to be disjoint from $A$, and $D$ is separating if and only if $A$ is separating in $Y$. Note that if $A$ is separating then the curves of $\partial A$ are parallel on $\partial Y$.

We wish to investigate the possible separating annuli $A$ of $K^{\prime} \cap Y$. Let $p$ : $\partial N(K) \rightarrow K$ be the covering projection and let $j: \partial N(K) \rightarrow \partial N(K)$ be the covering transformation, where $\partial N(K)$ is viewed as the orientable double covering of $K$. The curves of $\partial A$ on $\partial Y=\partial N(K)$ can be chosen to project via $p$ to simple closed curves on $K$. Without loss of generality, after an isotopy each component of $\partial A$ can be assumed to satisfy $C \cap j C=\varnothing$. There are 3 possibilities for $C$ :

(a) $C$ separates $\partial Y$ and $C$ is parallel to $j C$ on $\partial Y$.

(b) $C$ does not separate $\partial Y$ and $C$ is parallel to $j C$ on $\partial Y$.

(c) $C$ is not parallel to $j C$ on $\partial Y$.

These conditions have equivalent formulations as:

$\left(\mathrm{a}^{\prime}\right) p(C)$ separates $K$ into a punctured torus and a Möbius band.

$\left(\mathrm{b}^{\prime}\right) p(C)$ separates $K$ into a punctured Klein bottle and a Möbius band.

(c') $p(C)$ is a 2-sided nonseparating curve on $K$.

( $\alpha$ ) Suppose $A$ is a separating annulus of $K^{\prime} \cap Y$ with curves of $\partial A$ of type (a). Then $A$ must be parallel into $\partial Y$, since a boundary compression of $A$ yields a disk with a contractible boundary on $\partial Y$. Hence $A$ can be eliminated.

( $\beta$ ) Assume there is a separating annulus $A$ of $K^{\prime} \cap Y$, with loops of $\partial A$ in class (b) and $A$ is not parallel into $\partial Y$. It is easy to see that there is an annulus $A_{0}$ properly embedded in $N(K)$ with $\partial A_{0}=\partial A$ and $A_{0} \cap K=p(C)$, for $C$ a component of $\partial A$. Clearly $A \cup A_{0}$ separates $M$ and so is a torus $T$.

Let $V, W$ be the closures of the components of $M-T$. Then without loss of generality, $K \cap V$ is a Möbius band and $K \cap W$ is a punctured Klein bottle, since $K \cap T=p(C)$. Now $V$ is the union of 2 solid tori; they are the closure of a component of $Y-A$ and of $N(K)-A_{0}$. These 2 solid tori intersect along the annulus $A_{1}=\partial Y \cap V$. Because $A$ is not parallel into $\partial Y$, we observe that $A_{1}$ is not parallel to $A$. Also since $K \cap V$ is a Möbius band, it is easy to see that $A_{1}$ is not parallel to $A_{0}$.

Consequently $V$ can be fibered as a Seifert manifold with two exceptional fibres and the disk as orbit surface. So as $M$ is atoroidal, $T$ must compress in $W$ to give a 2-sphere $S . S$ bounds a ball $B^{3}$, since $M$ is irreducible, and $V \subset B^{3}$ is impossible because the centre-line of $K \cap V$ gives a nonzero element of $H_{1}\left(M, Z_{2}\right)$. Therefore $B^{3} \subset W, W$ is a solid torus, and the Seifert fibering of $V$ extends over $M$. Note that $M$ is thus a Seifert manifold as in (1) of $\S 2$.

Now $K \cap K^{\prime}$ must contain a curve parallel to $p(C)$ on $K$, since $K^{\prime}$ meets $\partial N(K)$ in the 2 components of $\partial A$ which are both parallel to $C$, and one of these 2 loops must be in an annulus of $K^{\prime} \cap N(K)$. Because $K-K^{\prime}$ is orientable, $K \cap K^{\prime}$ must include a component which is a centre of the Möbius band $K \cap V$. Therefore $K^{\prime} \cap N(K)$ has to contain a Möbius band with the same centre curve as that of $K \cap V$. 
By isotopically shrinking $V$, we can assume that $K^{\prime} \cap V$ has one component which is a Möbius band, and all the other components are annuli which are not boundary parallel in $V$. But then $K^{\prime} \cap W$ consists of a punctured Klein bottle plus some annuli. By an isotopy of $K^{\prime}$ all these latter annuli which are boundary parallel in the solid torus $W$ may be eliminated. Hence $K^{\prime} \cap V$ is a Möbius band which has a common centre-line with the Möbius band $K \cap V$. It is easy to find a further isotopy of $K^{\prime}$ so that $K^{\prime} \cap V=K \cap V$.

It now follows that $K^{\prime} \cap W$ and $K \cap W$ are both punctured Klein bottles in the solid torus $W$ with the same boundary curve. By the argument in Theorem 12 of [8], it follows that $K \cap W$ and $K^{\prime} \cap W$ are isotopic in $W$. So we have established that $K$ and $K^{\prime}$ are isotopic, which implies Theorem 5.

$(\gamma)$ Suppose there are separating annuli $A_{1}$ and $A_{2}$ of $K^{\prime} \cap Y$ such that neither $A_{1}$ nor $A_{2}$ is parallel into $\partial Y$, the curves of $\partial A_{i}, i=1,2$, are all of type (c) and the loops of $\partial A_{1}$ are parallel on $\partial Y$ to the components of $j\left(\partial A_{2}\right)$.

Let $C_{1}$ and $C_{2}$ be the curves of $\partial A_{1}$. Then after an isotopy of $A_{2}$, we can assume that the components of $\partial A_{2}$ are $j\left(C_{1}\right)$ and $j\left(C_{2}\right)$. As in $(\beta)$, there are disjoint annuli $A_{3}$ and $A_{4}$ properly embedded in $N(K)$ with $\partial A_{3}=C_{1} \cup j\left(C_{1}\right), \partial A_{4}=C_{2} \cup j\left(C_{2}\right)$ and $A_{3} \cap K=p\left(C_{1}\right), A_{4} \cap K=p\left(C_{2}\right)$. It is easy to see that $T=\cup_{i=1}^{4} A_{i}$ separates $M$ and so is a torus. Let $V, W$ be the closures of the components of $M-T$.

Assume without loss of generality that $K \cap V$ is the annulus in $K$ with boundary $p\left(C_{1}\right) \cup p\left(C_{2}\right)$. Then $K \cap W$ is a Möbius band with one hole. Clearly $K \cap V$ separates $V$ into 2 solid tori and since $A_{1}$ and $A_{2}$ are not parallel into $\partial Y$, it follows that $V$ can be fibered as a Seifert manifold with a disk as orbit surface and 2 exceptional fibres.

Again since $M$ is atoroidal and irreducible, $T$ must compress in $W$ to form a 2-sphere $S$ which bounds a ball $B^{3}$ in $M$. Suppose that $V \subset$ int $B^{3}$. By a small isotopy of $S$, we may assume that $S$ intersects $K$ transversely, without affecting $V \subset$ int $B^{3}$. All the simple closed curves of $S \cap K$ bound disks in $K$, because $K$ is incompressible. Let $\bar{D}$ denote the union of all these disks. Clearly $\bar{D}$ is a disjoint union of disks in $K$. Also $\partial \bar{D} \subset S \cap K$ and $K \cap V \subset K \cap$ int $B^{3}$ are disjoint. Therefore since $K \cap V$ is an annulus with noncontractible boundary curves in $K$, we see that $\bar{D} \subset K \cap W$. But then the connected surface $K-$ int $\bar{D}$ must lie in int $B^{3}$, as $K$ - int $\bar{D}$ contains $K \cap V$ and is disjoint from $S$. This contradicts our assumption that $K$ is incompressible. We conclude that $W \subset B^{3}$ and so $W$ is a solid torus.

If we perform a boundary compression of $K \cap W$ in $W$, then the result is a Möbius band with a contractible boundary curve on $T=\partial W$. Therefore $K$ is compressible, which is a contradiction. So we conclude that this case cannot occur. The analysis of the separating annuli of $K^{\prime} \cap Y$ is complete.

Suppose that $K^{\prime} \cap Y$ contains a component which is a nonseparating annulus $A$. Since a boundary compression of $A$ yields a disjoint nonseparating meridian disk, we see that the curves of $\partial A$ are not parallel on $\partial Y$ and both are nonseparating. So the components of $\partial A$ must be of type (b) or (c). 
(I) Assume $K^{\prime} \cap Y$ includes a nonseparating annulus $A$ with $\partial A=C_{1} \cup C_{2}$, where both $C_{1}$ and $C_{2}$ are of type (b). Then there are disjoint Möbius bands $J_{1}$ and $J_{2}$ which are properly embedded in $N(K)$, where $\partial J_{i}=C_{i}$ and $J_{i} \cap K$ is a centre-line for $J_{i}$, for $i=1,2$. So $L=J_{1} \cup A \cup J_{2}$ is an embedded Klein bottle in $M$.

Let $N(L)$ be a small neighborhood of $L$ in $M$, chosen so that $K \cap N(L)$ consists of 2 Möbius bands which have common centre-lines with $J_{1}$ and $J_{2}$. Let $W=M-$ int $N(L)$. Since $M$ is irreducible and atoroidal, $W$ is a solid torus. But $K \cap W$ is a Möbius band with a hole. We obtain the same contradiction as in $(\gamma)$ above. So this case cannot happen.

(II) Suppose there is a nonseparating annulus $A$ in $K^{\prime} \cap Y$ such that the components $C_{1}$ and $C_{2}$ of $\partial A$ are of type (c). Since $C_{1}$ is not parallel to $C_{2}$, we can perform an isotopy of $A$ so that $\partial A=C \cup j(C)$.

Exactly as in $(\gamma)$ above, there is an annulus $A_{0}$ properly embedded in $N(K)$ with $A_{0} \cap K=p(C)$ and $\partial A_{0}=\partial A$. If $A \cup A_{0}$ is a torus $T$, then clearly $T$ is nonseparating in $M$. $T$ cannot be incompressible since $M$ is atoroidal. However a compression of $T$ yields a nonseparating 2-sphere which contradicts the assumption that $M$ is irreducible.

Therefore $A \cup A_{0}=L$ must be a Klein bottle. But then exactly the same argument as in (I) above gives a contradiction. We conclude that this type of annulus cannot occur in $K^{\prime} \cap Y$.

To complete the proof of Theorem 5, assume first that $K^{\prime} \cap Y$ has a component which is a punctured torus $T_{0}$. Clearly $\partial T_{0}$ is of type (a). Hence all the curves of $K^{\prime} \cap \partial Y$ are either in classes (a) or (c). By (II), $K^{\prime} \cap Y$ has no nonseparating annuli. Hence by connectivity of $K^{\prime}$, we see that $K^{\prime} \cap \partial Y$ has no curves of type (c). By $(\alpha)$, any separating annulus of $K^{\prime} \cap Y$ is boundary parallel. So $K^{\prime}$ is isotopic to $K^{\prime \prime}$ with $K^{\prime \prime} \cap Y=T_{0}$ and $K^{\prime \prime} \cap K=C$, a single curve. Clearly $K^{\prime \prime}-C$ and $K-C$ are both orientable.

Next suppose that $K^{\prime} \cap Y$ has a component $E$ which is a sphere with 3 holes, and all the curves of $\partial E$ are of type (b). By $(\beta)$ and (I), it follows that $K^{\prime} \cap Y$ can contain only boundary parallel annuli. So after an isotopy, we may assume that $K^{\prime} \cap Y=E$.

Clearly no pair of the curves $C_{i}, i=1,2,3$, of $\partial E$ are parallel on $\partial Y$. A boundary compression of $E$ in $Y$ is given by isotoping $E$ along a disk $D$, which satisfies $\partial D=\lambda \cup \mu$ with $\lambda=D \cap E, \mu=D \cap \partial Y$ and $\lambda$ is not homotopic in $E$ keeping its ends fixed into $\partial E$. Such boundary compressions may be found e.g. by intersecting $E$ with the meridian disks of $Y$.

Suppose that the arc $\lambda$ of the boundary compression joins 2 different components of $\partial E$, say $C_{1}$ and $C_{2}$. Then after the isotopy of $E$ along $D$, we are left with a separating annulus $A$ in $Y$ with $\partial A$ consisting of curves parallel to $C_{3}$. By $(\beta)$, we may assume that $A$ is parallel into $\partial Y$. But this implies that $E$ is boundary parallel and so $K^{\prime}$ is isotopic to $K$ (see Lemma 15 of [8]).

Assume that the arc $\lambda$ has its ends on the same curve of $\partial E$, say $C_{3}$. Then after the isotopy of the boundary compression, the result is 2 separating annuli $A_{1}$ and $A_{2}$ in 
$Y$ with $\partial A_{i}$ having components parallel to $C_{i}$, for $i=1,2$. Again by $(\beta)$, both $A_{1}$ and $A_{2}$ can be supposed boundary parallel and hence $K^{\prime}$ is isotopic to $K$.

(†) Suppose that $K^{\prime} \cap Y$ has a component $E$ which is a sphere with 3 holes such that one of the curves of $\partial E$ is of type (a) and 2 are of type (c). By (II), $K^{\prime} \cap Y$ has no nonseparating annuli. The curves of $K^{\prime} \cap \partial Y$ of type (c) are all parallel to either $C$ or $j(C)$, where $C$ is some fixed loop of type (c). Clearly every component of $K^{\prime} \cap K$ which is 2 -sided and nonseparating gives rise to 2 curves of type (c) in $K^{\prime} \cap \partial Y$, one parallel to $C$ and one parallel to $j(C)$. So there are equal numbers of such curves.

Suppose both of the curves of $\partial E$ of type (c) are parallel to $C$. By $(\gamma)$ and ( $\alpha)$, we may assume that $K^{\prime} \cap Y$ has exactly 2 components, $E$ and $A$, where $A$ is a separating annulus with both curves of $\partial A$ parallel to $j(C)$.

We now look at the first boundary compression of the system $K^{\prime} \cap Y$. If $A$ is boundary compressed, the result is a separating meridian disk for $Y$ disjoint from $E$. Hence $E$ is contained in a solid torus and so $E$ is compressible. This is a contradiction and hence $E$ must be boundary compressed first.

Exactly as before, there are two possible results for the effect of the boundary compression of $E$. We obtain from $E$ either a single separating annulus $A_{0}$ or two separating annuli $A_{1}$ and $A_{2}$. In either case, all the components of $\partial A_{0}, \partial A_{1}$ and $\partial A_{2}$ are parallel to $C$ or of type (a). By $(\alpha)$ and $(\gamma)$, we see that these annuli must be boundary parallel and so $E$ is boundary parallel. After the isotopy of $K^{\prime}$ to $K^{\prime \prime}$ taking $E$ to $\partial Y$ and then past $K$, it follows that $K^{\prime \prime} \cap K=C$ as desired.

Assume $\partial E$ has one curve parallel to each of $C$ and $j(C)$. By $(\gamma)$ and $(\alpha)$, we may suppose that $K^{\prime} \cap Y=E$. But then $K^{\prime}$ and $K$ intersect in 2 curves only, $C_{1}$ and $C_{2}$, where $C_{1}=p(C)$ is 2-sided and nonseparating on $K$ and $K-C_{2}$ is a punctured torus. Let $C_{3}$ be a simple closed curve in $K-C_{2}$ which intersects $C_{1}$ transversely in one point. Since $C_{3}$ is 2-sided on $K$, the intersection number mod 2 of the homology classes $\left|C_{3}\right| \in H_{1}\left(M, Z_{2}\right)$ and $|K| \in H_{2}\left(M, Z_{2}\right)$ is zero. But $C_{3}$ crosses $K^{\prime}$ at one point so the intersection number $\bmod 2$ of $\left|C_{3}\right|$ and $\left|K^{\prime}\right|$ is one. This contradicts $|K|=\left|K^{\prime}\right| \cdot(\dagger)$

Assume finally that there is a component $E$ of $K^{\prime} \cap Y$ which is a sphere with 3 holes and one of the curves of $\partial E$ is of type (b) and 2 are of type (c). There is a loop $C$ on $\partial Y$ of type (c) such that all of the type (c) curves of $K^{\prime} \cap \partial Y$ are parallel to $C$ or to $j(C)$ on $\partial Y$. Exactly as above, there are equal numbers of curves parallel to $C$ and to $j(C)$.

Suppose that among the nonboundary parallel annuli of $K^{\prime} \cap Y$, there are boundary curves parallel to $C, j(C)$ and of type (b). The first boundary compression of one of these annuli yields a meridian disk with boundary curve $C^{\prime}$ disjoint from all the boundary curves of the annuli. But then it follows immediately that $C^{\prime} \cap$ $j\left(C^{\prime}\right)=\varnothing$ can be assumed, without loss of generality. So $p\left(C^{\prime}\right)$ is a 2-sided simple closed curve on $K$ which bounds a disk $D$ with $D \cap K=p\left(C^{\prime}\right)$. But $p\left(C^{\prime}\right)$ is noncontractible on $K$ and this implies that $K$ is compressible, a contradiction. 
Clearly there are at most 3 types of intersection curves of $K$ and $K^{\prime} ; 2$-sided nonseparating curves parallel to $p(C)$, a single one-sided curve $C_{0}$ with the property that $K-C_{0}$ is an open punctured Klein bottle and possibly some parallel 2-sided curves which separate $K$ into a punctured Klein bottle and a Möbius band with centre-line $C_{0}$. It is easy to find a simple closed curve $C^{\prime}$ which is one-sided in $K$, crosses $p(C)$ transversely at one point and misses $C_{0}$. Without loss of generality $C^{\prime}$ intersects each of the components of $K \cap K^{\prime}$ which are parallel to $p(C)$ at one point and is disjoint from the other curves of $K \cap K^{\prime}$.

Suppose $K \cap K^{\prime}$ has an even number of curves parallel to $p(C)$. Then the intersection number mod 2 of $\left|C^{\prime}\right|$ and $\left|K^{\prime}\right|$ is zero. However $C^{\prime}$ is one-sided on $K$ so the intersection number $\bmod 2$ of $\left|C^{\prime}\right|$ and $|K|$ is one. This gives a contradiction and therefore $K \cap K^{\prime}$ has an odd number of components parallel to $p(C)$.

Assume that none of the annuli of $K^{\prime} \cap Y$ has a boundary curve of type (b). Then by (II), $K \cap K^{\prime}$ has no separating annuli and by $(\gamma)$ we may suppose that all the separating annuli have boundary components parallel to $C$. If $\partial E$ has one curve parallel to each of $C$ and $j(C)$ then there are no annuli in $K^{\prime} \cap Y$. If $\partial E$ has both components parallel to $C$ say, then there must be exactly one annulus $A$ in $K^{\prime} \cap Y$ with the loops of $\partial A$ parallel to $j(C)$. But then $K \cap K^{\prime}$ includes 2 curves parallel to $p(C)$, which is impossible. So we conclude there are no annuli in $K^{\prime} \cap Y$.

On the other hand, suppose that no boundary curve of an annulus of $K^{\prime} \cap \partial Y$ is parallel to $C$ say. If $\partial E$ has one curve parallel to each of $C$ and $j(C)$, then there are again no annuli in $K^{\prime} \cap Y$. On the other hand if $\partial E$ has both curves parallel to $C$ then there must be exactly two curves of $K^{\prime} \cap \partial Y$ parallel to $j(C)$. Once more we obtain that $K \cap K^{\prime}$ has 2 loops parallel to $p(C)$, a contradiction. Therefore the only possibility is that $K^{\prime} \cap Y=E$ and $\partial E$ has 3 nonparallel curves.

Finally a boundary compression of $\partial E$ yields one or 2 separating annuli with boundary curves parallel to $C, j(C)$ or of type (b). By $(\beta)$ we may assume the latter possibility does not occur. Also by $(\gamma)$, we cannot then obtain 2 annuli unless one of them is boundary parallel. So the end result is that $K^{\prime} \cap Y$ is a single separating annulus with boundary loops parallel to $C$ say.

Now it is straightforward to isotope $K^{\prime}$ and $K$ together until $K^{\prime} \cap K=K-$ int $A$ is an annulus with centre-line $p(C)$. Then $K^{\prime} \cap K$ is a Möbius band with one puncture and so there are 2 isotopy classes of choices of a one-sided curve on $K^{\prime} \cap K$. For suitable selection, we obtain a one-sided loop $C_{1} \subset K^{\prime} \cap K$ which satisfies $K-C_{1}$ and $K^{\prime}-C_{1}$ are both orientable. Lastly there is clearly a small isotopy of $K^{\prime}$ to $K^{\prime \prime}$ so that $K^{\prime \prime} \cap K=C_{1}$ and this finishes the proof of Theorem 5.

REMARK. If $K \cap K^{\prime \prime}=C$ as in Theorem 5, then $K^{\prime \prime} \cap Y$ is a punctured torus $T_{0}$. The disk of some boundary compression of $T_{0}$ in $Y$ can be extended readily to a disk $D$ with the property that $\partial D=\lambda \cup \mu$, with $D \cap K^{\prime \prime}=\lambda$ and $D \cap K=\mu$. Hence $\lambda$ is not homotopic in $K^{\prime \prime}$ keeping its ends fixed into $C$, and similarly for $\mu$ in $K$.

Let $K^{*}$ denote the result of an isotopy of $K^{\prime \prime}$ taking a neighborhood of $\lambda \cup C$ in $K^{\prime \prime}$ onto a neighborhood of $\mu \cup C$ in $K$, using $D$. Then $K^{*} \cap K=K-$ int $A=K^{*}$ - int $A^{*}$, where $A, A^{*}$ are annuli in $K, K^{*}$ respectively. In general $A^{*}$ need not be 
parallel to $A$, although $A \cup A^{*}$ bounds a solid torus. So it appears unlikely that $K^{*}$ is always isotopic to $K$, but we are unable to resolve this problem (see §7).

5. Non-Haken 3-manifolds. We wish to examine the situation in Theorem 5 when $K^{\prime}$ is also part of a one-sided Heegaard decomposition of $M$. This will be the case when $M$ is non-Haken.

THEOREM 6. Suppose $M \neq R P^{3}$ is a closed, irreducible atoroidal 3-manifold which has a one-sided Heegaard decomposition of genus $3, M=N(K) \cup Y$.

(A) There are at most 3 one-sided Heegaard splittings of genus 3, $M=N(K) \cup Y$ $=N\left(K^{\prime}\right) \cup Y^{\prime}=N\left(K^{\prime \prime}\right) \cup Y^{\prime \prime}$, such that $|K|=\left|K^{\prime}\right|=\left|K^{\prime \prime}\right|$ and if $M=N\left(K^{*}\right) \cup$ $Y^{*}$ is another such decomposition with $\left|K^{*}\right|=|K|$, then $K^{*}$ is isotopic to one of $K, K^{\prime}$ or $K^{\prime \prime}$.

(B) Assume in addition that $M$ is non-Haken. Then there are at most 3 embeddings $K, K^{\prime}, K^{\prime \prime} \subset M$ such that $|K|=\left|K^{\prime}\right|=\left|K^{\prime \prime}\right|$ and if $K^{*} \subset M$ is another embedding with $\left|K^{*}\right|=|K|$, then $K^{*}$ is isotopic to either $K, K^{\prime}$ or $K^{\prime \prime}$.

In either (A) or (B), the embeddings $K^{\prime}, K^{\prime \prime} \subset M$ can be chosen so that $K \cap K^{\prime}=K$ $\cap K^{\prime \prime}=K^{\prime} \cap K^{\prime \prime}=C$, a single curve.

REMARKs. (a) Exactly as in the remark at the end of $\S 4$, our methods are insufficient to decide if the embeddings $K, K^{\prime}, K^{\prime \prime} \subset M$ are isotopic to each other. However, this seems unlikely to be true in general (see §7).

(b) In Theorem 6(B), the hypothesis that $M$ is non-Haken can be replaced by the weaker assumption that $M$ contains no separating incompressible surfaces of genus 2. By Lemma 2 of [8], the latter supposition guarantees that any embedding of $K$ in $M$ gives rise to a one-sided Heegaard splitting of genus 3, i.e. $M=N(K) \cup Y$.

Proof. As at the beginning of the proof of Theorem 5, since $M$ is assumed irreducible, non-Haken and $\neq R P^{3}$ in Theorem 6(B), it follows that any embedding of $K$ in $M$ is incompressible. Also by Lemma 2 of [8], any embedding of $K$ in $M$ yields a one-sided Heegaard splitting of genus 3 of $M$.

By Theorem 5, if $M=N(K) \cup Y$ and $K^{\prime} \subset M$ with $|K|=\left|K^{\prime}\right|$ then after an isotopy of $K^{\prime}$, we can achieve $K \cap K^{\prime}=C$, where $K-C$ and $K^{\prime}-C$ are both orientable. By the remark at the end of $\S 4$, there is a disk $D$ embedded in $M$ such that $\partial D=\lambda \cup \mu$, where $\lambda=D \cap K^{\prime}, \mu=D \cap K$ and $\mu$ is not homotopic in $K$ keeping its ends fixed into $C$. We will assume without loss of generality that $K^{\prime}$ is not isotopic to $K$. Note that $M-\left(K \cup K^{\prime}\right)$ has 2 components; we will denote the closures of these components by $Y_{1}$ and $Y_{2}$, where $D \subset Y_{1}$.

Suppose now that $K^{\prime \prime}$ is another embedded genus 3 nonorientable surface in $M$ with $\left|K^{\prime \prime}\right|=|K|$, and $K^{\prime \prime}$ is not isotopic to $K$ or $K^{\prime}$. By Theorem 5, after an isotopy of $K^{\prime \prime}$ we can achieve that $K \cap K^{\prime \prime}$ is a single curve $C_{0}$ and $K-C_{0}, K^{\prime \prime}-C_{0}$ are both orientable. By Lemma $3, C_{0}$ and $C$ are isotopic in $K$. So by a further isotopy of $K^{\prime \prime}$, we may assume that $K^{\prime \prime} \cap K=C$. The main step in the proof of Theorem 6 is to find an isotopy of $K^{\prime \prime}$ so that $K^{\prime \prime} \cap K^{\prime}=C$ is attained, but $K^{\prime \prime} \cap K=C$ remains unaltered by this isotopy. 
Without loss of generality, $K^{\prime \prime}$ intersects $D$ transversely except possibly at the 2 points of $\partial \lambda=\partial \mu=D \cap C$. By the incompressibility of $K^{\prime \prime}$, an isotopy of $K^{\prime \prime}$ can be performed which eliminates the entire set of simple closed curves of $K^{\prime \prime} \cap D$. The remaining arcs of $K^{\prime \prime} \cap D$ all have both endpoints on $\lambda$, since $K^{\prime \prime} \cap K=C$ implies $K^{\prime \prime} \cap \mu=\partial \mu$. Consequently there is an isotopy of $K^{\prime \prime}$ which is obtained by pushing all the arcs of $K^{\prime \prime} \cap D$ down along $D$ past $K^{\prime}$. After the isotopy, it follows that $K^{\prime \prime} \cap D=D \cap C=\partial \mu$ and $K^{\prime \prime} \cap K=C$ continues to be true.

Let $N(C \cup \lambda)$ be a small neighborhood of $C \cup \lambda$ in $K^{\prime}$. Because $K^{\prime \prime} \cap$ int $\lambda=\varnothing$, $N(C \cup \lambda)$ can be chosen so that $K^{\prime \prime} \cap N(C \cup \lambda)=C$. But $K^{\prime}-$ int $N(C \cup \lambda)$ is an annulus. Therefore all the curves of $K^{\prime \prime} \cap \partial N\left(K^{\prime}\right)$ are now of type (c), except for one of type (a) (as in the proof of Theorem 5). Here, by the assumptions in (A) or (B) of Theorem 6, we can find a splitting $M=N\left(K^{\prime}\right) \cup Y^{\prime}$. If there are no components of $K^{\prime \prime} \cap \partial N\left(K^{\prime}\right)$ of type (c), then $K^{\prime \prime} \cap K^{\prime}=C$ has been achieved as desired.

Therefore it suffices to assume that $K^{\prime \prime} \cap Y^{\prime}$ contains some annuli with boundary curves of type (c) and a component $E$ which is a sphere with 3 holes. Clearly all the boundary parallel annuli of $K^{\prime \prime} \cap Y^{\prime}$ in $Y^{\prime}$ can be eliminated by an isotopy of $K^{\prime \prime}$ without affecting $K^{\prime \prime} \cap K=C$. By the argument (†) in the proof of Theorem 5, since $K^{\prime \prime}$ is assumed to be not isotopic to $K^{\prime}$, the only possibility is that $K^{\prime \prime} \cap Y=E$ $\cup A$ where $A$ is an annulus and $E$ must be parallel into $\partial Y^{\prime}$.

It is easy to see that the isotopy of $K^{\prime \prime}$ which takes $E$ into $\partial Y^{\prime}$ and then past $K^{\prime}$ can be performed so that $K^{\prime \prime} \cap K=C$ continues to hold. The effect of this isotopy is to eliminate the pair of 2-sided curves in $K^{\prime \prime} \cap K^{\prime}$. Hence after the isotopy, we have attained $K^{\prime \prime} \cap K^{\prime}=C$ as desired. So $K \cap K^{\prime}=K^{\prime \prime} \cap K=K^{\prime} \cap K^{\prime \prime}=C$ is now the case. Note that $K^{\prime \prime} \subset Y_{2}$.

If $K^{*} \subset M$ is an embedding with $K^{*} \cap K=K^{*} \cap K^{\prime}=K \cap K^{\prime}=C$, then there are 2 possibilities: $K^{*} \subset Y_{1}$ or $K^{*} \subset Y_{2}$. We prove next that if $K^{*} \subset Y_{1}$ then $K^{*}$ is isotopic to $K$ or to $K^{\prime}$. Since $K \cap K^{\prime}=K^{*} \cap K=K^{*} \cap K^{\prime}=C$, it follows immediately that $K^{*} \cap \partial D=C \cap \partial D=\partial \lambda$. So assuming that $K^{*}$ crosses $D$ transversely, we find that $K^{*} \cap D$ consists of an arc $\gamma$ with $\partial \lambda=\partial \gamma$ plus some simple closed curves, since $D \subset Y_{1}$. Because $K^{*}$ is incompressible, we can perform an isotopy of $K^{*}$ in $Y_{1}$ which removes all the loops of $K^{*} \cap D$.

As $D \cap K^{*}=\gamma$, simultaneous isotopies can be performed of $K^{\prime}$ and $K^{*}$ taking neighborhoods of $\lambda \cup C$ and $\gamma \cup C$ respectively on $K^{\prime}$ and $K^{*}$ along $D$ onto a neighborhood of $\mu \cup C$ in $K$. After the isotopy, we obtain that $K^{*} \cap K=K^{\prime} \cap K$ $=N(C \cup \mu)$. Hence $\operatorname{cl}\left(K^{*}-K\right)=A^{*}, \operatorname{cl}\left(K^{\prime}-K\right)=A^{\prime}$ and $K-\operatorname{int} N(C \cup \mu)=$ $A$ are all annuli with common boundaries.

Since $K^{\prime}$ is not isotopic to $K$, it follows that $A \cup A^{\prime}$ bounds a solid torus $W$, but $A$ and $A^{\prime}$ are not parallel annuli in $W$. On the other hand, clearly $A^{*} \subset W$ and so $A^{*}$ is parallel into $\partial W$ (any incompressible annulus properly embedded in a solid torus is boundary parallel). We conclude that $A^{*}$ must be parallel to either $A^{\prime}$ or $A$. Hence $K^{*}$ is isotopic to $K^{\prime}$ or to $K$, as asserted.

By the argument achieving $K^{\prime \prime} \cap K^{\prime}=K^{\prime \prime} \cap K=K \cap K^{\prime}=C$, it follows that $K^{\prime \prime} \subset Y_{2}$. Clearly $K^{\prime \prime}$ separates $Y_{2}$ into 2 components; let $X_{1}$ and $X_{2}$ denote the closures of the components of $Y_{2}-K^{\prime \prime}$. Now the closures of the components of 
$M-\left(K \cup K^{\prime \prime}\right)$ are obviously $X_{2} \cup Y_{1}$ and $X_{1}$, where we assume that $K \subset X_{1}$ and $K^{\prime} \subset X_{2}$ without loss of generality. By the remark at the end of $\S 4$, there is a disk $D_{1}$ in either $X_{2} \cup Y_{1}$ or in $X_{1}$ with $\partial D_{1}=\lambda_{1} \cup \mu_{1}$, where $\lambda_{1}=D_{1} \cap K^{\prime \prime}, \mu_{1}=D_{1} \cap K$ and $\lambda_{1}$ is not homotopic in $K^{\prime \prime}$ keeping its ends fixed into $C$.

Suppose $D_{1} \subset X_{2} \cup Y_{1}$. Then the argument above shows that $K^{\prime}$ is isotopic to $K$ or to $K^{\prime \prime}$, since $K^{\prime}$ lies in the closure of the component of $M-\left(K \cup K^{\prime \prime}\right)$ which contains $D_{1}$. Hence $D_{1} \subset X_{1}$ must be the case. Now by the assumptions in (A) or (B) of Theorem 6, there is a one-sided Heegaard decomposition $M=N\left(K^{\prime}\right) \cup Y^{\prime}$. Clearly the closures of the components of $M-\left(K^{\prime} \cup K^{\prime \prime}\right)$ are $X_{2}$ and $X_{1} \cup Y_{1}$, since $K \subset X_{1}$ and $K^{\prime} \subset X_{2}$. Using $K^{\prime}$ and $K^{\prime \prime}$ in the roles of $K$ and $K^{\prime \prime}$, we find that there is a disk $D_{2}$ in $X_{2}$ or in $X_{1} \cup Y_{1}$, such that $\partial D_{2}=\lambda_{2} \cup \mu_{2}$, where $\lambda_{2}=D_{2} \cap K^{\prime \prime}$, $\mu_{2}=D_{2} \cap K^{\prime}$ and $\lambda_{2}$ is not homotopic in $K^{\prime \prime}$ keeping its ends fixed into $C$.

If $D_{2} \subset X_{1} \cup Y_{1}$ then we again reach the contradiction that $K$ is isotopic to $K^{\prime}$ or to $K^{\prime \prime}$. Hence $D_{2} \subset X_{2}$ and we have attained the symmetrical situation that each of the regions $Y_{1}, X_{1}$ and $X_{2}$ contains one of the disks $D, D_{1}$ and $D_{2}$ respectively.

To complete the proof, suppose that $K^{*}$ is an arbitrary embedding of the genus 3 nonorientable surface in $M$ with $\left|K^{*}\right|=|K|$. By the preceding method, we can achieve that $K \cap K^{*}=K^{\prime} \cap K^{*}=C$, after a suitable isotopy of $K^{*}$. Also without loss of generality $K^{*} \subset X_{1} \cup X_{2}=Y_{2}$, since if $K^{*} \subset Y_{1}$ then $K^{*}$ is isotopic to $K$ or to $K^{\prime}$. As usual, by the hypotheses in (A) or (B) in Theorem 6, we can write $M=N\left(K^{\prime \prime}\right) \cup Y^{\prime \prime}$.

Exactly as before, we can perform an isotopy of $K^{*}$ in $X_{1} \cup X_{2}$ which eliminates all the intersection points of $K^{*}$ and $D_{1}$ except for the 2 points $D_{1} \cap C=\partial \lambda_{1}=\partial \mu_{1}$, without altering $K^{*} \cap K=K^{*} \cap K^{\prime}=C$. So $K^{*}$ intersects $\partial N\left(K^{\prime \prime}\right)$ in one curve of type (a) and all the remaining loops are of type (c). By the argument denoted by $(\dagger)$ in the proof of Theorem 5, it follows that after the boundary parallel annuli of $K^{*} \cap Y^{\prime \prime}$ have been removed, then $K^{*} \cap Y^{\prime \prime}=E \cup A$ where $E$ is a sphere with 3 holes and $A$ is an annulus. Also $E$ is boundary parallel in $Y^{\prime \prime}$. Clearly the isotopy of $E$ into $\partial Y^{\prime \prime}$ actually occurs in $X_{1} \cup X_{2}$.

Consequently we can isotope $K^{*}$ in $X_{1} \cup X_{2}$ to shift $E$ into $\partial Y^{\prime \prime}$ and then past $K^{\prime \prime}$. This attains $K^{\prime \prime} \cap K^{*}=C$, without affecting $K^{*} \cap K=K^{*} \cap K^{\prime}=C$. But then $K^{*}$ must lie in $X_{1}$ or in $X_{2}$. This implies that $K^{*}$ is isotopic to one of $K, K^{\prime}$ or $K^{\prime \prime}$ and the proof of Theorem 6 is finished.

RemarKs. (a) Let $M=N(K) \cup Y=N\left(K^{\prime}\right) \cup Y^{\prime}$ with $|K|=\left|K^{\prime}\right|$ and $K \cap K^{\prime}=$ $C$, where $M$ is as in Theorem 6. Let $Y_{1}, Y_{2}$ be the closures of the components of $M-\left(K \cup K^{\prime}\right)$. Assume that there are disks $\bar{D}_{i} \subset Y_{i}$ with $\partial \bar{D}_{i}=\bar{\lambda}_{i} \cup \bar{\mu}_{i}$, where $\bar{\lambda}_{i}=\bar{D}_{i} \cap K, \bar{\mu}_{i}=\bar{D}_{i} \cap K^{\prime}$ and $\bar{\lambda}_{i}$ is not homotopic in $K$ into $C$ keeping its ends fixed, for $i=1,2$.

Then it is easy to see by the techniques in the proof of Theorem 6, if $K^{*} \subset M$ is any embedding of the genus 3 nonorientable surface in $M$ with $|K|=\left|K^{*}\right|$, then $K^{*}$ is isotopic to $K$ or $K^{\prime}$. Therefore there are at most 2 isotopy classes of embeddings of this surface, $K, K^{\prime} \subset M$, realising the particular element $|K|$ of $H_{2}\left(M, Z_{2}\right)$.

Returning to the notation of the proof of Theorem 6, suppose that for example $\lambda_{2}=\lambda_{1}$ (recall that $\lambda_{j}=D_{j} \cap K^{\prime \prime}$ for $j=1,2$ ). Then $D_{1} \cup D_{2}=D^{\prime}$ is a disk 
properly embedded in $Y_{2}=X_{1} \cup X_{2}$, such that $\partial D^{\prime}=\mu_{1} \cup \mu_{2}$ with $D^{\prime} \cap K=\mu_{1}$, $D^{\prime} \cap K^{\prime}=\mu_{2}$. So the disks $\bar{D}_{1}, \bar{D}_{2}$ above can be chosen to be $D, D^{\prime}$ and there are at most 2 isotopy classes of embeddings. Note that $K^{\prime \prime}$ must be isotopic to $K$ or to $K^{\prime}$ in this case.

(b) It is not difficult to construct 3-manifolds $M=N(K) \cup Y$ which have embedded surfaces $K, K^{\prime}, K^{\prime \prime}$ in a symmetrical configuration as in the proof of Theorem 6, i.e. $M=N\left(K^{\prime}\right) \cup Y^{\prime}=N\left(K^{\prime \prime}\right) \cup Y^{\prime \prime}$ and $K \cap K^{\prime}=K \cap K^{\prime \prime}=K^{\prime} \cap$ $K^{\prime \prime}=C$. Also the 3 closures of the components of $M-\left(K \cup K^{\prime} \cup K^{\prime \prime}\right)$ all possess properly embedded disks as in the proof of Theorem 6, but $K^{\prime} \cap Y$ is not boundary parallel in $Y$, and similarly for $K^{\prime \prime} \cap Y$ in $Y$ and $K^{\prime \prime} \cap Y^{\prime}$ in $Y^{\prime}$. It is not clear whether or not $K, K^{\prime}$ and $K^{\prime \prime}$ are isotopic to one another, although certainly the obvious way of achieving this fails (see §7).

\section{Unique isotopy class of embeddings.}

THEOREM 7. Let $M$ be a 3-manifold in one of the classes in \$2. By Lemma 2, M has a one-sided Heegaard decomposition of genus 3, $M=N(K) \cup Y$. Assume that $K^{\prime} \subset M$ is an embedding of the genus 3 nonorientable surface with $|K|=\left|K^{\prime}\right|$. Then $K^{\prime}$ is isotopic to $K$.

Proof. By Theorem 5, it suffices to assume that $K \cap K^{\prime}=C$, where $K-C$ and $K^{\prime}-C$ are both orientable. We consider separately the cases when $M$ is in the class of examples (1), (2) and (3) in $§ 2$.

(1) Let $N_{1}$ be a small fibered regular neighborhood of the exceptional fibre of multiplicity 2 in $M$. As in the proof of Lemma 2, we may assume that $N_{1} \cap K$ is a Möbius band $J$. Let $C_{1}$ be a centre-line of $J$. A simple closed curve $C_{2}$ in $K$ can be found so that $C_{2}$ intersects $C_{1}$ transversely in one point and $K-C_{2}$ is orientable. By Lemma $3, C_{2}$ is isotopic to $C$ in $K$. Hence we may suppose without loss of generality that $C_{1}$ and $C$ cross transversely at exactly one point. Then since $K^{\prime} \cap K=C$, by choosing $N_{1}$ sufficiently small and observing that $C_{1}$ is a core circle of $N_{1}$, we can obtain that $K^{\prime}$ intersects $N_{1}$ transversely and $K^{\prime} \cap N_{1}$ is a single meridian disk $D_{1}$ for $N_{1}$.

Let $A$ be an annulus of ordinary fibres in $M$ with $A \cap N_{1}=\partial A$, such that there is one exceptional fibre in each of the components of $M-N_{1}-A$. Let $N(A)$ be a small regular neighborhood of $A$ in $M$ and assume that $N(A) \cap N_{1}$ consists of 2 annuli in $\partial N(A) \cap \partial N_{1}$. Then the closures of the components of $M-N_{1}-N(A)$ are solid tori $N_{2}$ and $N_{3}$ which are fibered regular neighborhoods of the exceptional fibres of multiplicity $4 k$ and $m$ respectively.

Since $N_{1}$ is a fibered neighborhood of the multiplicity 2 exceptional fibre, any ordinary fibre in $\partial N_{1}$ can be assumed to meet $\partial D_{1}$ in exactly 2 points. Hence $\partial A \cap \partial D_{1}$ has 4 points, 2 in each component of $\partial A$. Supposing that $K^{\prime}$ intersects $A$ transversely, we find that $K^{\prime} \cap A$ has only 2 components $\lambda_{1}$ and $\lambda_{2}$ which are arcs. By an isotopy, we may assume $K^{\prime} \cap A$ has no contractible loops.

Let $\partial A=C_{3} \cup C_{4}$. It is easy to see that the 2 points $C_{3} \cap \partial D_{1}$ are separated by the 2 points $C_{4} \cap \partial D_{1}$ in $\partial D_{1}$, since $C_{3}$ and $C_{4}$ are parallel simple closed curves in 
$\partial N_{1}$. Suppose $\lambda_{1}$ (and $\left.\lambda_{2}\right)$ has one end on $C_{3}$ and one on $C_{4}$. Then $K^{\prime} \cap\left(N_{1} \cup\right.$ $N(A))=D_{1} \cup N\left(\lambda_{1}\right) \cup N\left(\lambda_{2}\right)$ where $N\left(\lambda_{i}\right)$ is a small regular neighborhood of $\lambda_{i}$ in $K^{\prime}$, without loss of generality. Note that the points of $\partial \lambda_{1}$ do not separate $\partial \lambda_{2}$ on $\partial D_{1}$. Let $E=D_{1} \cup N\left(\lambda_{1}\right) \cup N\left(\lambda_{2}\right)$ and let $X=N_{1} \cup N(A)$. There are 3 possibilities for the surface $E$, as shown in Figure 1. By transversality of the intersection of $K^{\prime}$ and $A$, the points $x, y$ as indicated in Figure 1 must lie in different components of $\partial X=\partial N_{2} \cup \partial N_{3}$. However in cases (b) and (c) in Figure 1, there are paths in $\partial E$ from $x$ to $y$, which is a contradiction since $\partial E \subset \partial X$. We conclude that (a) is the only possibility.

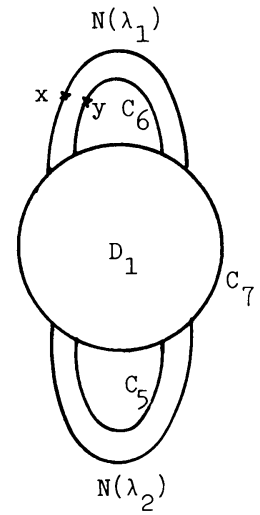

(a)

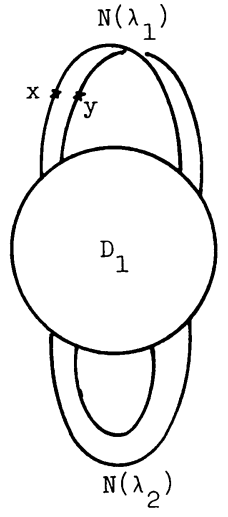

(b)

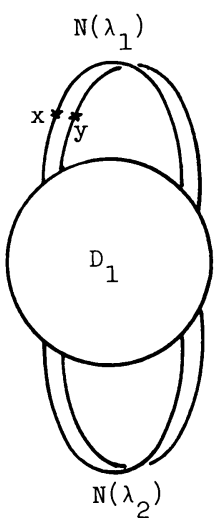

(c)

FIGURE 1

Let $C_{5}, C_{6}, C_{7}$ denote the components of $\partial E$, as shown in Figure 1. Since the 2 ordinary fibres of $\partial A$ intersect $\partial D_{1}$ in $\partial \lambda_{1} \cup \partial \lambda_{2}$, it follows that ordinary fibres in $\partial N_{1}$ can be found which cross $C_{5}, C_{6}$ and $C_{7}$ transversely in one, one and 2 points respectively. Also one of the components of $\partial X$ contains $C_{5}$ and $C_{6}$, while the other component of $\partial X$ includes $C_{7}$. Assume therefore that $C_{5} \cup C_{6}$ lies in $\partial N_{i}$, for $i=2$ or 3 .

If $C_{5}$ and $C_{6}$ bound meridian disks in $N_{i}$, then the multiplicity of the exceptional fibre in $N_{i}$ must be one. Hence $m=1, i=3$ and $M$ is a lens space. In this case, Theorem 7 follows by Theorem 12 of [8]. The only other possibility is that $K^{\prime} \cap N_{i}$ is a connected surface bounded by $C_{5} \cup C_{6}$. In this case a boundary compression of $K^{\prime} \cap N_{i}$ in $N_{i}$ yields a surface with a single contractible boundary curve. By incompressibility of $K^{\prime}$, this implies that $K^{\prime} \cap N_{i}$ must be a boundary parallel disk, after the boundary compression. Therefore $K^{\prime} \cap N_{i}=\varnothing$ can be achieved by a further isotopy of $K^{\prime}$. Since a core-circle of $N_{2}$ is the exceptional fibre of multiplicity 
$4 k$, such a core-circle has intersection number $1 \bmod 2$ with $K$ and with $K^{\prime}$, because $|K|=\left|K^{\prime}\right|$. We conclude that $K^{\prime} \cap N_{3}=\varnothing$ holds.

Then $K^{\prime} \subset N_{1} \cup N_{2}$ may be assumed. Since $N_{1} \cap N_{2}$ is an annulus of ordinary fibres in $\partial N_{1} \cap \partial N_{2}$, it is easy to show that $K^{\prime} \cap N_{1}$ must have one component a Möbius band plus all other components annuli. The desired conclusion that $K^{\prime}$ is isotopic to $K$ now follows exactly as in case $(\beta)$ in the proof of Theorem 5 .

Finally suppose that the arc $\lambda_{1}$ of $K^{\prime} \cap A$ has both ends on $C_{3}$ and therefore $\partial \lambda_{2} \subset C_{4}$. Then one of the closures of the components of $A-\lambda_{1}$ is a disk $D . D$ may be used for a boundary compression of $K^{\prime} \cap\left(M-\operatorname{int} N_{1}\right)$ in $M-\operatorname{int} N_{1}$. Let $K^{\prime \prime}$ denote the result of the isotopy of the boundary compression applied to $K^{\prime}$. Then $K^{\prime \prime} \cap N_{1}$ is a Möbius band (cf. the proof of Lemma 10 in [8]). Note that if $\partial D=\lambda_{1} \cup \mu$, where $\mu \subset C_{3}$, then $\lambda_{1}$ has its endpoints on opposite sides of $C_{3}$ in $\partial N_{1}$. So $K^{\prime \prime} \cap \partial N_{1}$ is easily seen to be disjoint from $C_{3}$, i.e. $K^{\prime \prime} \cap \partial N_{1}$ and $C_{3}$ are parallel curves on $\partial N_{1}$.

We can now assume that $K^{\prime \prime} \cap A$ consists of simple closed noncontractible loops only, since $K^{\prime \prime} \cap \partial A=\varnothing$ may be achieved by a further isotopy. Therefore $K^{\prime \prime} \cap \partial N_{3}$ consists of ordinary fibres. Exactly the same argument as above shows that all the components of $K^{\prime \prime} \cap N_{3}$ must be boundary parallel annuli. So $K^{\prime \prime} \cap N_{3}=\varnothing$ can be obtained and then $K^{\prime \prime}$ can be isotoped onto $K$.

(2) Let $N_{i}, i=1,2,3$, be small fibered regular neighborhoods of the 3 exceptional fibres in $M$. As in the proof of Lemma 2, it may be supposed that $N_{i} \cap K$ is a Möbius band denoted $J_{i}$, for $i=1,2,3$. A simple closed curve $C_{0}$ in $K$ can be found such that $K-C_{0}$ is orientable and $C_{0}$ intersects a centre-line of $J_{i}$ transversely in exactly one point, for all $i$. By Lemma 3, we can isotope $K^{\prime}$ so that $K^{\prime} \cap K=C_{0}$. By choosing the neighborhoods $N_{i}$ sufficiently small, we can therefore achieve that $K^{\prime} \cap N_{i}$ is a single meridian disk $D_{i}$ for $N_{i}$, for each $i=1,2,3$.

Let $W=M-\cup_{i}$ int $N_{i}$. Then $K^{\prime} \cap W$ is a genus 3 nonorientable surface with 3 holes, which we denote by $K_{0}^{\prime}$. There is a homeomorphism $\phi: W \rightarrow E \times S^{1}$, where $E$ is a sphere with 3 holes. Let $T_{i}=\phi\left(\partial N_{i}\right)$, let $C_{i}=\phi^{-1}\left(E \times\{1\} \cap T_{i}\right)$ and let $h_{i}$ be an ordinary fibre in $\partial N_{i}$ which intersects $C_{i}$ transversely at a single point, for $i=1,2,3$. We may assume without loss of generality that $\phi(K \cap W)=E \times\{1\}$ as well, so $C_{i}=\partial J_{i}$. Now since $K^{\prime} \cap \partial N_{i}$ is a meridian curve $C_{i}^{\prime}$ for $N_{i}$ and $N_{i}$ is a regular neighborhood of an exceptional fibre with invariants $(r, \pm 2),(s, \pm 2)$ or $(t, \pm 2)$, we may suppose without loss of generality that $C_{i}^{\prime}$ intersects $C_{i}$ transversely in exactly 2 points, for all $i$.

Let $F$ denote the surface $\phi\left(K_{0}^{\prime}\right)$ in $E \times S^{1}$. We investigate the projection mapping from $E \times S^{1} \rightarrow S^{1}$ restricted to $F$, which we denote by $p$. By a small isotopy of $K^{\prime}$, it may be supposed that $p$ is a Morse function on $F$. Because $C_{i}^{\prime} \cap C_{i}$ has 2 points, it can be assumed that $E \times\{z\} \cap \partial F$ has exactly 6 points for each $z$ in $S^{1}$. Therefore the level set $p^{-1}(z)=E \times\{z\} \cap F$ consists of 3 arcs plus some simple closed curves, when $z$ is not a critical value of $p$.

Assume first of all that for every $z$ in $S^{1}$ which is not a critical value of $p$, each arc of the level set $p^{-1}(z)$ runs between $T_{i}$ and $T_{j}$ for $i \neq j$. A critical point of $p$ is either a local maximum, minimum or a saddle (where $S^{1}$ is locally parametrized by $\arg z$ ). 
Suppose there is a saddle with a critical value $z_{0}=\exp i \theta_{0}$, such that $2 \operatorname{arcs}$ of the level set $p^{-1}\left(\exp i\left(\theta_{0}-\varepsilon\right)\right)$ coalesce in $p^{-1}\left(z_{0}\right)$, where $\varepsilon>0$ is arbitrarily small. Since the 3 arcs of $p^{-1}\left(\exp i\left(\theta_{0}-\varepsilon\right)\right)$ have ends on different boundary components of $E \times\left\{\exp i\left(\theta_{0}-\varepsilon\right)\right\}$, it is easy to see that in $p^{-1}\left(\exp i\left(\theta_{0}+\varepsilon\right)\right)$ one of the arcs has both ends on the same curve of $\partial E \times\left\{\exp i\left(\theta_{0}+\varepsilon\right)\right\}$ (see Figure 2). This is a contradiction to our hypothesis, since $\exp i\left(\theta_{0}+\varepsilon\right)$ is not a critical value for $\varepsilon$ small.
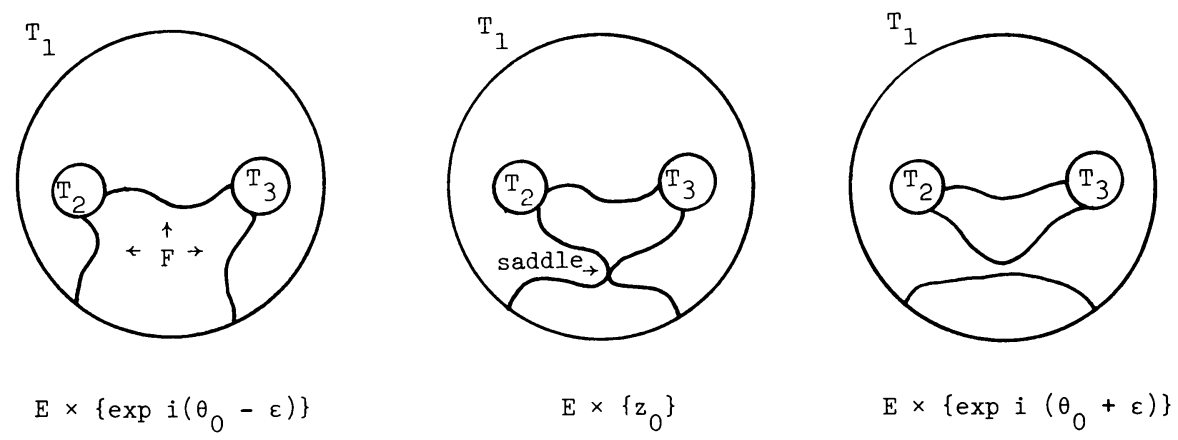

FIGURE 2

Consequently, if every arc of $p^{-1}(z)$ runs from $T_{i}$ to $T_{j}$ for $i \neq j$, where $z$ is any noncritical value of $p$, then there are no saddles involving 2 arcs. So each saddle must be between 2 loops or between an arc and a loop. By the usual Morse theory methods (see [6]), all the local maxima and minima can be cancelled in pairs with saddles, and we end up with saddles only. Let us consider the "birth" and "death" of a loop component in the level sets (see Figure 3). For a birth, $\arg z_{0}<\arg z_{1}<\arg z_{2}$, while for a death, $\arg z_{0}>\arg z_{1}>\arg z_{2}$ in Figure 3.

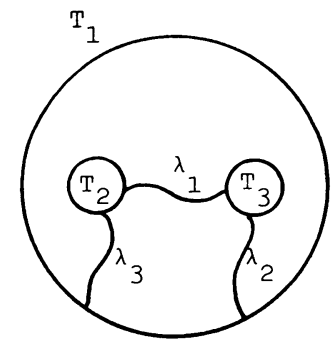

$\mathrm{E} \times\left\{\mathrm{z}_{0}\right\} \underset{\mathrm{\text {Death }}}{\stackrel{\text { Birth }}{\longleftrightarrow}}$

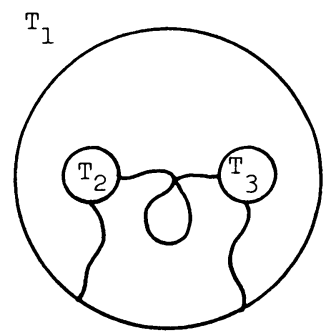

$\mathrm{E} \times\left\{\mathrm{z}_{1}\right\} \underset{\text { Death }}{\stackrel{\text { Birth }}{\rightleftarrows}}$

\section{FIGURE 3}

For $z$ a noncritical value of $p$, let us denote an arc of $p^{-1}(z)$ by $\lambda_{i}$, where $\lambda_{i}$ runs from $T_{j}$ to $T_{k}$ and $\{i, j, k\}=\{1,2,3\}$. There are 2 possibilities for the successive birth and death of a loop component in the level sets. Either (a) the birth and death both occur at $\operatorname{arcs} \lambda_{i}, \lambda_{i}^{\prime}$ running from $T_{j}$ to $T_{k}$ or (b) the birth is at an $\operatorname{arc} \lambda_{i}$ with ends on $T_{j}$ and $T_{k}$ while the death is at an $\operatorname{arc} \lambda_{j}^{\prime}$ running from $T_{i}$ to $T_{k}$ say. 
In case (a), the birth and death clearly give an orientable handle of $F$. But the loop $C$ formed in a level set $p^{-1}(z)$ between the birth and death bounds a disk in $E \times\{z\}$ and is nonseparating in $F$ (the loop $C$ is a cocore of the handle). This contradicts the fact that $F$ is incompressible in $E \times S^{1}$. Hence case (a) cannot occur.

In case (b), let $C$ denote the loop formed in some level set between the birth and death, which occur at $\operatorname{arcs} \lambda_{i}, \lambda_{j}^{\prime}$ respectively. It is easy to see that there is an arc in $F$ which starts at $\partial \lambda_{i} \cap T_{k}$, ends at $\partial \lambda_{j}^{\prime} \cap T_{k}$ and crosses $C$ at one point. This arc can be closed up by an arc in $F \cap T_{k}$ to give a loop meeting $C$ transversely in one point. So $C$ is nonseparating in $F$, and we reach the same contradiction as in (a). Therefore we find that there are no births and deaths, and $p$ has no critical points. This implies that $F$ is a disjoint union of 3 annuli, which is a contradiction (cf. [5] for a very similar argument).

Consequently for some $z_{0}$ in $S^{1}$ which is not a critical value of $p$, there is an $\operatorname{arc} \lambda$ of $p^{-1}\left(z_{0}\right)$ which has both endpoints on $T_{3}$ say. There are 2 possibilities for $\lambda$ in $E \times\left\{z_{0}\right\}$ : either $\lambda$ is parallel into $\partial E \times\left\{z_{0}\right\}$ or else $\lambda$ separates $E \times\left\{z_{0}\right\}$ into 2 annuli. Suppose the latter is true and let $\mu, \gamma$ be the other 2 arcs of $p^{-1}\left(z_{0}\right)$. Since $p^{-1}\left(z_{0}\right) \cap T_{3}$ has only 2 points, we see that $\partial \mu \subset T_{1}$ and $\partial \gamma \subset T_{2}$ without loss of generality. Also $\mu$ and $\gamma$ are both parallel into $\partial E \times\left\{z_{0}\right\}$. Consequently after replacing $\lambda$ by $\mu$ if necessary, we may assume that there is a disk $D$ in $E \times\left\{z_{0}\right\}$ with $\partial D=\lambda \cup \nu$, where $\nu \subset T_{3}, \lambda$ is an arc of $p^{-1}\left(z_{0}\right)$ and $z_{0}$ is not a critical value of $p$.

We now perform a boundary compression of $F$ in $E \times S^{1}$ using $D$. If $F^{\prime}$ is the new surface in $E \times S^{1}$ after this compression, then $E \times\left\{z_{0}\right\} \cap F^{\prime}$ has only 2 arcs. Consequently $F^{\prime} \cap T_{3}$ is disjoint from $E \times\left\{z_{0}\right\} \cap T_{3}=\phi\left(C_{3}\right)$ and we see that the arc $\nu$ must run from one side of the curve $F \cap T_{3}$ to the other. Thus the boundary compression of $F$ to $F^{\prime}$ pushes one of the Möbius bands in $F$ across $T_{3}$ (cf. Lemma 10 in [8]). Applying the same boundary compression to $K_{0}^{\prime}$ in $W\left(v^{\prime a} \phi^{-1}\right)$, we obtain an isotopy of $K^{\prime}$ to $K^{\prime \prime}$ so that $K^{\prime \prime} \cap N_{3}$ is a Möbius band and $K^{\prime \prime} \cap N_{i}$ is a single meridian disk, for $i=1,2$. Since $\phi\left(K^{\prime \prime} \cap W\right)=F^{\prime}$, it follows that $K^{\prime \prime} \cap \partial N_{3}$ is disjoint from the loop $C_{3}$. Hence the curve $K^{\prime \prime} \cap \partial N_{3}$ is isotopic in $\partial N_{3}$ to $K \cap \partial N_{3}=C_{3}$. Therefore by an isotopy of $K^{\prime \prime}$, we can arrange that $K^{\prime \prime} \cap N_{3}=K \cap$ $N_{3}=J_{3}$.

The next step is to boundary compress $K^{\prime \prime} \cap W$ in $W$. Instead of using the above method with the Morse function $p$, which becomes much more complicated due to the presence of noncontractible loops in the level sets, we employ a more direct argument. Let $A_{1}, A_{2} \subset W$ be disjoint annuli of ordinary fibres with $\partial A_{1}=h_{1} \cup h_{3}$ and $\partial A_{2}=h_{2} \cup h_{4}$, where $h_{3}, h_{4} \subset \partial N_{3}, h_{1} \subset \partial N_{1}$ and $h_{2} \subset \partial N_{2}$. We may assume also that $h_{3}, h_{4}$ both intersect $C_{3}$ transversely at one point, and similarly for the pairs of curves $h_{2}, C_{2}$ and $h_{3}, C_{4}$. We denote $K^{\prime \prime} \cap W$ by $G$.

Suppose that $G$ intersects $A_{1}$ and $A_{2}$ transversely. Since $G \cap \partial N_{3}=C_{3}$, it follows that there is a single point in $G \cap A_{i} \cap \partial N_{3}$, for $i=1,2$. Consequently $G \cap A_{i}$ has exactly one arc running from $\partial N_{i}$ to $\partial N_{3}$, and all the other arcs of $G \cap A_{i}$ have both ends on $\partial N_{i}$, for $i=1$ or 2 . However for $i=1$ or $2, G \cap \partial N_{i}=C_{i}^{\prime}$ and $A_{i} \cap \partial N_{i}=h_{i}$, where $C_{i}^{\prime}$ is a meridian curve for $N_{i}$. Since $N_{i}$ is a regular neighborhood of an 
exceptional fibre with invariants $(x, \pm 2)$, where $x=r, s$ or $t$ and $r, s, t \geqslant 3$, it follows that $C_{i}^{\prime}$ and $h_{i}$ intersect in $x \geqslant 3$ points. Therefore we can find $\operatorname{arcs} \lambda_{i}$ in $G \cap A_{i}$ with both ends on $\partial N_{i}$, for $i=1$ and 2 .

Let $D_{i}$ be the closure of a component of $A_{i}-\lambda_{i}$ which is a disk. Then we can use $D_{i}$ to perform boundary compressions of $G$ across $\partial N_{i}$, for $i=1$ and 2. Let $G^{*}$ be the new surface in $W$ after these two compressions of $G$. Because there are fewer intersections of $h_{i}=A_{i} \cap \partial N_{i}$ with $G^{*}$ than with $G$, it follows as before that the boundary compressions must push Möbius bands in $G$ across $\partial N_{i}$ (cf. Lemma 10 in [8]), for $i=1$ or 2 . Therefore applying the compressions to $K^{\prime \prime}$, we obtain an isotopy of $K^{\prime \prime}$ to $K^{*}$, such that $K^{*} \cap N_{3}=K^{\prime \prime} \cap N_{3}=J_{3}$ remains true and $K^{*} \cap N_{i}$ is a Möbius band for $i=1,2$.

Now $G^{*}$ is clearly a sphere with 3 holes with one boundary curve on each of $\partial N_{i}$, for $i=1,2,3$. So it immediately follows that $G^{*}$ is incompressible and boundary incompressible in $W$. Therefore after isotopies of $A_{1}$ and $A_{2}$, we can arrange that $G^{*} \cap A_{i}$ is a single arc $\gamma_{i}$ running from $\partial N_{i}$ to $\partial N_{3}$, for $i=1,2$. [In fact if $\mu$ is an arc of $G^{*} \cap A_{i}$ with both ends on $\partial N_{i}$, then since $G^{*}$ is boundary incompressible, there is a disk $D$ in $G^{*}$ with $\partial D=\mu \cup \nu$ where $\nu \subset \partial G^{*}$. If $D^{\prime}$ is the closure of a component of $A_{i}-\mu$ which is a disk, then it is easy to find an isotopy of $A_{i}$ taking $D^{\prime}$ past $D$ and so eliminating $\mu$.]

The endpoint of $\gamma_{i}$ on $\partial N_{3}$ lies in $K \cap W$ since $G^{*} \cap \partial N_{3}=C_{3}=K \cap \partial N_{3}$. So we may assume without loss of generality that there is a nontrivial subarc $\delta_{i}$ of $\gamma_{i}$ at this endpoint which is contained in $K \cap W$. Let $\mu_{i}=\gamma_{i}-$ int $\delta_{i}$, and let $N\left(\mu_{i}\right)$ be a small regular neighborhood of $\mu_{i}$ in $W$. Since $\mu_{i}$ is an arc with an end on $N_{i}$, clearly there is an isotopy $H_{t}$ of $M$ expanding $N_{i}$ so that $H_{1}\left(N_{i}\right)=N_{i} \cup N\left(\mu_{i}\right)$, for $i=1$ and 2. $H_{t}$ can be chosen so that $H_{t}$ is the identity on $N_{1}, H_{1}\left(\gamma_{i}\right)=\delta_{i}$ and $K \cap H_{1}\left(A_{i}\right)=K^{*} \cap$ $H_{1}\left(A_{i}\right)=\delta_{i}$, for $i=1$ or 2 . Therefore if we relabel $H_{1}(W)$ as $W, H_{1}\left(N_{i}\right)$ as $N_{i}$ and $H_{1}\left(A_{i}\right)$ as $A_{i}$, we obtain that $K^{*} \cap N_{3}=K \cap N_{3}=J_{3}$ is still true and $K^{*} \cap A_{i}=K$ $\cap A_{i}=\delta_{i}$, for $i=1,2$.

Finally the curve $C_{i}^{*}=K^{*} \cap \partial N_{i}$, for $i=1$ or 2 , has the properties that $C_{i}^{*}$ intersects the ordinary fibre $h_{i}$ in $\partial A_{i}$ transversely in one point and $C_{i}^{*}$ bounds a Möbius band in $N_{i}$. Since any two simple closed curves in $\partial N_{i}$ having these two properties are isotopic, we find that $C_{1}^{*}$ is isotopic to $C_{i}$. So we may achieve by further isotopies of $K^{*}$ first that $K^{*} \cap \partial N_{i}=K \cap \partial N_{i}=C_{i}$ and then that $K^{*} \cap N_{i}$ $=K \cap N_{i}=J_{i}$, for $i=1$ and 2 .

But these isotopies can be chosen so that $K^{*} \cap A_{i}=K \cap A_{i}=\delta_{i}$ remains true, for $i=1,2$. Hence we have achieved that $K^{*}-K$ is contained in the open disk $K^{*}-\cup_{i} J_{i}-\delta_{1}-\delta_{2}$. Therefore it is now straightforward to isotope $K^{*}$ onto $K$.

(3) Assume that there is a disk $D$ satisfying condition (*) in $\S 2$, i.e. $\partial D \cap C^{\prime}$ has 2 points, where $C^{\prime}$ is the lift of $C$ to $\partial N(K)$. Then if $K^{\prime}$ intersects $D$ transversely, it follows that $K^{\prime} \cap D$ has one arc plus some simple closed curves, as $K^{\prime} \cap \partial N(K)=C^{\prime}$ may be supposed. Since $K^{\prime}$ is incompressible, by an isotopy of $K^{\prime}$ keeping $K^{\prime} \cap N(K)$ fixed, we can obtain that $K^{\prime} \cap D$ is a single $\operatorname{arc} \lambda$. 
Let $K^{\prime} \cap Y$ be denoted by $T_{0}$, which is a punctured torus. Suppose there is a disk $D^{\prime}$ in $T_{0}$ with $\partial D^{\prime}=\lambda \cup \mu$ where $\mu \subset \partial T_{0}$. Let $N(D)$ and $N\left(D^{\prime}\right)$ be small regular neighborhoods of $D$ and $D^{\prime}$ in $Y$. Then $\partial\left(N(D) \cup N\left(D^{\prime}\right)\right)$ has 3 disk components, one of which is parallel to $D$. Both of the other disks are disjoint from $T_{0}$ and it is easy to see that they cannot both be parallel into $\partial Y$. But this implies that $T_{0}$ lies in the complement of a meridian disk in $Y$, i.e. in a solid torus. This contradicts the fact that $T_{0}$ is incompressible. Hence $\lambda$ is not parallel into $\partial T_{0}$ in $T_{0}$.

Let $D_{1}$ be another meridian disk for $Y$, chosen so that $D, D_{1}$ are disjoint and $Y-D-D_{1}$ is simply connected. We may suppose that $T_{0}$ intersects $D_{1}$ transversely in arcs only. Assume that $T_{0} \cap D_{1}$ has an arc $\gamma$ which is parallel to $\lambda$ in $T_{0}$. Let $D_{2}$ be the disk in $T_{0}$ with the property that $\partial D_{2}$ is contained in $\lambda \cup \gamma \cup \partial T_{0}$. Without loss of generality, $\gamma$ and $D_{2}$ can be chosen so that $D_{1} \cap$ int $D_{2}=\varnothing$. Let $N\left(D \cup D_{1} \cup D_{2}\right)$ be a small regular neighborhood of $D \cup D_{1} \cup D_{2}$ in $Y$. There are 4 disk components in $\partial N\left(D \cup D_{1} \cup D_{2}\right)$. Let $D_{3}$ be the unique one of these 4 disks which is not parallel to $D$ or into $\partial T_{0}$ and which can be deformed by a small isotopy to intersect $T_{0}$ transversely in fewer arcs than $D_{1}$.

We now replace $D_{1}$ by $D_{3}$. Since $D_{3} \cap T_{0}$ has fewer components than $D_{1} \cap T_{0}$, by induction we eventually find a meridian disk $D_{4}$ which is disjoint from $D$, satisfies $Y-D-D_{4}$ is simply connected and intersects $T_{0}$ transversely in arcs only, none of which are parallel to $\lambda$ on $T_{0}$. We can also assume that none of the $\operatorname{arcs}$ in $T_{0} \cap D_{4}$ are parallel into $\partial T_{0}$, by the same method as applied to $D$.

Let $\mu$ be an arc of $T_{0} \cap D_{4}$, chosen so that there is a subdisk $D_{0}$ of $D_{4}$ with $\partial D_{0}$ contained in $\mu \cup \partial D_{4}$ and $T_{0} \cap$ int $D_{0}=\varnothing$. We can employ $D_{0}$ to do a boundary compression of $T_{0}$ in $Y$, since $\mu$ is not parallel into $\partial T_{0}$. This gives an isotopy of $K^{\prime}$ to $K^{\prime \prime}$ so that $K^{\prime \prime} \cap Y$ is an annulus $A$. Note that $A \cap D=T_{0} \cap D=\lambda$, and $\lambda$ has ends on different components of $\partial A$ because $\lambda$ is not parallel to $\mu$ in $T_{0}$.

The closures of the components of $D-\lambda$ are subdisks $D^{\prime}$ and $D^{\prime \prime}$ of $D$. Since $A$ is a separating annulus in $Y$, there is an annulus $A_{1}$ in $\partial Y$ with $\partial A_{1}=\partial A$. We may assume that $\partial D^{\prime}=\lambda \cup \delta$, where $\delta \subset A_{1}$. Hence if $A$ is boundary compressed using $D^{\prime}$, we obtain a disk with contractible boundary in $\partial Y$. Consequently this disk and $A$ are both parallel into $\partial Y$, and we conclude that there is an isotopy between $K^{\prime}$ and $K$.

\section{Conclusion.}

1. We make some observations concerning the homeotopy group $\mathcal{H}(M)$, when $M$ is an irreducible, atoroidal 3-manifold which has a genus 3 one-sided Heegaard splitting $M=N(K) \cup Y$. Let $\{h\} \in \mathcal{H}(M)$ denote the isotopy class of a homeomorphism $h: M \rightarrow M$.

Let $G$ be the kernel of the representation $\mathcal{H}(M) \rightarrow$ Aut $H_{1}\left(M, Z_{2}\right)$. A homeomorphism $h: M \rightarrow M$ satisfies $\{h\} \in G$ if and only if $h_{*}: H_{1}\left(M, Z_{2}\right) \rightarrow h_{1}\left(M, Z_{2}\right)$ is the identity. Then by Poincare duality, $h_{*}: H_{2}\left(M, Z_{2}\right) \rightarrow H_{2}\left(M, Z_{2}\right)$ is the identity and $h_{*}(|K|)=|K|$. By Theorem 5 after an isotopy of $h$ we can obtain that $h(K)$ and $K$ intersect transversely at a single curve $C$, so that $K-C$ and $h(K)-C$ are both orientable. 
In addition, as $h(K)$ also determines a one-sided Heegaard decomposition of $M$, i.e. $M=N(h(K)) \cup h(Y)$ where $N(h(K))=h(N(K))$, we may apply Theorem 6(A). Consequently there are at most 3 homeomorphisms $h_{i}: M \rightarrow M$ with the properties that $\left\{h_{i}\right\} \in G$ and if $h$ is any homeomorphism from $M$ to $M$ with $\{h\} \in G$, then $h(K)$ is isotopic to $h_{i}(K)$, for $i=1,2$ or 3 . Note that the mappings $h_{i}$ can be chosen so that $h_{1}$ is the identity and $K \cap h_{2}(K)=K \cap h_{3}(K)=h_{2}(K) \cap$ $h_{3}(K)=C$, a single curve.

Therefore we have proved that the subgroup $G^{\prime}$ of $G$, which consists of isotopy classes of homeomorphisms mapping $K$ onto $K$, has index at most 3 in $G$. Since $G$ has very small index in $\mathcal{H}(M)$, if $G^{\prime}$ can be calculated then this gives excellent information about $\mathcal{H}(M)$. Note that by Corollary $4, \mathcal{H}(K) \cong \mathrm{GL}(2, Z)$. Let $G_{1}$ be the subgroup of $\mathcal{H}(K)$ consisting of elements $\{f\}$, where $f$ is a homeomorphism from $K$ to $K$ which extends to some homeomorphism $h: M \rightarrow M$. Let $G_{2}$ be the subgroup of $G_{1}$ which has members $\{f\}$ such that $f$ extends to a homeomorphism $h$ : $M \rightarrow M$ which is isotopic to the identity. Then clearly $G^{\prime} \cong G_{1} / G_{2}$. In [2], the groups $G_{1}, G_{2}, G^{\prime}, G$ and $\mathcal{H}(M)$ are computed for the examples (1) and (3) in $\S 2$.

2. In [3], examples are given of 3-manifolds $M$ which possess at least two inequivalent Heegaard splittings of genus 2, i.e. $M=X \cup X^{\prime}=Y \cup Y^{\prime}$, where $X \cap X^{\prime}=\partial X=\partial X^{\prime}, Y \cap Y^{\prime}=\partial Y=\partial Y^{\prime}$ and $X, X^{\prime}, Y, Y^{\prime}$ are all handlebodies of genus 2, but there is no homeomorphism $h: M \rightarrow M$ with $h(X)=Y$ or $Y^{\prime}$ and $h\left(X^{\prime}\right)=Y^{\prime}$ or $Y$. The 3-manifolds $M$ discussed in [3] are Seifert manifolds with $S^{2}$ as orbit surface and 3 exceptional fibres. Also these 3-manifolds are homology 3 -spheres, so are irreducible and non-Haken. No bound on the number of inequivalent Heegaard splittings (of genus 2) of a 3-manifold is known, in contrast to the result in Theorem 6(A) for one-sided Heegaard splittings of genus 3 .

If a 3-manifold $M$ has a one-sided Heegaard splitting of genus 3, $M=N(K) \cup Y$, then there is a (unique) 2-fold covering $p: \tilde{M} \rightarrow M$ with the property that $p^{-1}(K)=\tilde{K}$ is the orientable double covering of $K$ (cf. [8]). Then $\tilde{M}$ has a Heegaard splitting of genus 2, $\tilde{M}=X \cup X^{\prime}$ with $\partial X=\partial X^{\prime}=X \cap X^{\prime}=\tilde{K}$. If $g: \tilde{M} \rightarrow \tilde{M}$ is the covering transformation then $g(X)=X^{\prime}$. The results in [3] give some evidence that there may not always be a unique isotopy class of embeddings of $K$ in $M$ realising a particular class in $H_{2}\left(M, Z_{2}\right)$, as in Theorem 7 (see Remark (a) following Theorem 6).

\section{REFERENCES}

1. Joan S. Birman and D. Chillingworth, On the homeotopy group of a non-orientable surface, Proc. Cambridge Philos. Soc. 71 (1972), 437-448. MR 45 \# 9334.

2. Joan S. Birman and J. H. Rubinstein, Homeotopy groups of some non-Haken 3-manifolds (to appear).

3. Joan S. Birman, F. Gonzales-Acuna and J. M. Montesinos, Heegaard splittings of prime 3-manifolds are not unique, Michigan Math. J. 23 (1976), 97-103. MR 55 \# 4177.

4. G. Bredon and J. Wood, Non-orientable surfaces in orientable 3-manifolds, Invent. Math. 7 (1969), 83-110. MR 39 \# 7616.

5. M. Culler, W. Jaco and J. H. Rubinstein, J. London Math. Soc. (to appear).

6. J. Milnor, Lectures on the h-cobordism theorem, Math. Notes, Princeton Univ. Press, Princeton, N. J., 1965. MR 32 \#352.

7. P. Orlik, Seifert manifolds, Lecture Notes in Math., vol. 291, Springer-Verlag, Berlin and New York, 1972. MR 54 \# 13950. 
8. J. H. Rubinstein, One-sided Heegaard splittings of 3-manifolds, Pacific J. Math. 76 (1978), 185-200.

9. On 3-manifolds that have finite fundamental group and contain Klein bottles, Trans. Amer. Math. Soc. 251 (1979), 129-137.

10. W. Thurston, Geometry and topology of 3-manifolds, Math. Notes, Princeton Univ. Press, Princeton, N. J. (to appear).

11. F. Waldhausen, Gruppen mit Zentrum und 3-dimensionale Mannifaltigkeiten, Topology 6 (1967), 505-517. MR 38 \#5223.

12. __ On irreducible 3-manifolds which are sufficiently large, Ann. of Math. (2) 87 (1968), 56-88. MR 36 \# 7146.

Department of Mathematics, University of Melbourne, Parkville, Victoria 3052, Australia 